BNL-52492

FORMAL REPORT

\title{
RADIATION ENVIRONMENT AND SHIELDING FOR A HIGH LUMINOSITY COLLIDER DETECTOR
}

\author{
RECENLU \\ MAY 05 1990 \\ OSTI
}

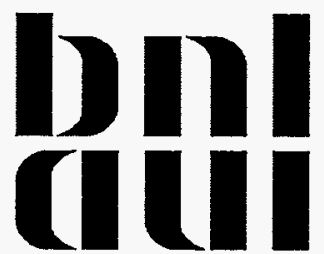

December 1995

\footnotetext{
BROOKHAVEN NATIONAL LABORATORY ASSOCIATED UNIVERSITIES, INC.

UNDER CONTRACT NO. DE-AC02-76CH00016 WITH THE UNITED STATES DEPARTMENT OF ENERGY
} 



\title{
RADIATION ENVIRONMENT AND SHIELDING FOR A HIGH LUMINOSITY COLLIDER DETECTOR
}

\author{
M.V. DIWAN ${ }^{a}$, Y. FISYAK ${ }^{b}$ N.V. MOKHOV \\ Superconducting Super Collider Laboratory*, Dallas, TX 75237 \\ D.M. LEE, G.H. SANDERS ${ }^{d}$, L. WATERS, W.B. WILSON \\ Los Alamos National Laboratory, Los Alamos, NM 87545 \\ B. MOORE \\ University of Mississippi, University, MS 38677 \\ M.D. MARX \\ State University of New York at Stony Brook, Stony Brook, NY 11794
}

C.R. WUEST

Lawrence Livermore National Laboratory, Livermore, CA 94551

J.P. RUTHERFOORD, C. ZEITNITZ'

University of Arizona, Tucson, AZ 85721

Yu. V. EFREMENKO

University of Tennessee, Knoxville, TN 37996

V.L. MORGUNOV

Institute for Theoretical and Experimental Physics (ITEP), Moscow, 117259 Russia

\section{A. SMIRNOV}

St. Petersburg Nuclear Physics Institute, Gatchina, Leningrad, Dist. 188350 Russia

* Operated by the Universities Research Association, Inc. for the U.S. Department of Energy under Contract No. DE-AC35-89ER40486

a Currently at Brookhaven National Laboratory, Upton, NY 11973

- Joint appointment at Moscow State University, Moscow, 177234 Russia. Currently at University of California at Davis, Davis, CA 95616

c Currently at FERMILAB, MS 345, Batavia, IL 60510

- Currently at California Institute of Technology, Pasadena, CA 91125

- Currently at 1711 23rd St. Niceville, FL 32578

1 Currently at Universitaet Mainz, Inst. fuer Physik, D-55099, Mainz, Germany 


\section{DISCLAIMER}

This report was prepared as an account of work sponsored by an agency of the United States Government. Neither the United States Government nor any agency thereof, nor any of their employees, nor any of their contractors, subcontractors, or their employees, makes any warranty, express or implied, or assumes any legal liability or responsibility for the accuracy, completeness, or usefulness of any information, apparatus, product, or process disclosed, or represents that its use would not infringe privately owned rights. Reference herein to any specific commercial product, process, or service by trade name, trademark, manufacturer, or otherwive, does not necessarily constitute or imply its endorsement, recommendation, or favoring by the United States Government or any agency, contractor or subcontractor thereof. The views and opinions of authors expressed herein do not necessarily state or reflect those of the United States Government or any agency, contractor or subcontractor thereof.

Printed in the United States of America

Available from

National Technical Information Service

U.S. Department of Commerce

5285 Port Royal Road

Springfield, VA 22161

NTIS price codes:

Printed Copy: A03; Microfiche Copy: A01 


\begin{abstract}
Detectors now under design for use in the proposed high energy high luminosity colliders must deal with unprecedented radiation levels. We have performed a comprehensive study for the GEM detector at the SSC to determine the best way to shield critical detector components from excessive radiation, with special attention paid to the low energy neutrons and photons. We have used several detailed Monte-Carlo simulations to calculate the particle fluxes in the detector. We describe these methods and demonstrate that two orders of magnitude reduction in the neutron and photon fluxes can be obtained with appropriate shielding of critical forward regions such as the low beta quadrupoles and the forward calorimeter.
\end{abstract}





\subsection{INTRODUCTION}

We have performed simulation studies of the neutron, photon, and charged particle fluxes for the proposed GEM detector [1] for the SSC. In this paper we describe our calculations of the neutron fluxes and the photon fluxes caused by neutron absorption. A detailed description of the radiation environment can be found in Reference [2]. During the process of these studies we have developed techniques for these types of simulations that will be useful for other collider detectors.

Historically neutrons have not caused significant hit rates in collider experiments at other accelerator laboratories, although extra hits have been measured in the forward muon chambers of the CDF and D0 detectors at the Fermilab Tevatron. Much higher radiation levels are expected at the SSC compared to all previous colliders because of the high center of mass energy ( $40 \mathrm{TeV})$ and the standard luminosity of $10^{33} \mathrm{~cm}^{-2} \mathrm{~s}^{-1}\left(10^{8} \mathrm{pp}\right.$ collisions per second with the beam current of $1.3 \times 10^{14}$ protons in each ring. A year of exposure at the SSC, SSCY, is defined to be $10^{7} \mathrm{~s}$ ). Therefore, careful consideration of shielding is needed, especially to assure low hit and trigger rates in the muon detectors surrounding the calorimeter. Though there is much discussion of the problem in previous reports [3-6], we believe this is the first detailed and comprehensive solution of the shielding problem in a realistic high luminosity detector for the SSC.

First, we briefly describe the design of the GEM detector. Then in Section 2 we describe the computational tools used for this study. In Section 3 we estimate the relative magnitudes of the various radiation sources in the detector. In Sections 4 and 5 detailed description of the shielding calculations is given.

\subsection{THE GEM DETECTOR}

Figure 1 is a schematic of the GEM detector. The detector is optimized for precision measurements of Gammas, Electrons, and Muons at high luminosities $\left(\sim 10^{34} \mathrm{~cm}^{-2} \mathrm{~s}^{-1}\right)$. The detailed description of the detector and the materials can be obtained from Reference [1], and was used in the simulations described in this paper. The detector can be divided in three parts:

1) The central detector cavity consists of a silicon tracker and interpolating cathode pad chambers. The outer radius of the cylindrical cavity is about $1 \mathrm{~m}$ and its total length is $4 \mathrm{~m}$. The main concern in this region of the detector is the total radiation dose to the sensitive detector elements such as the silicon tracker and a secondary concern is the random hit rates due to albedo (flux emitted backwards from the walls) 
neutrons and photons. A detailed description of the neutron and photon fluxes is given below. The occupancies due to these particles are shown to be less than $1 \%$ [1]. The dose from charged particles at standard luminosity to a detector placed $\mathrm{r} \mathrm{cm}$ from the beam line is $50 \mathrm{Mrad} /(\mathrm{r} / \mathrm{cm})^{2} / \mathrm{SSCY}$. The dose to the silicon due to neutrons can be significant if there is no shielding. The lifetime of silicon after exposure to neutrons with kinetic energy above $100 \mathrm{keV}$, the threshold where the displacement cross section increases by an order of magnitude, is known to be about $10^{14}$ neutrons $/ \mathrm{cm}^{2}$ [7].

2) The calorimeter consists of barrel, endcap, and forward sampling noble liquid calorimeters, covering the pseudorapidity intervals $|\eta|<1.3,1.3<|\eta|<3.0$, and $3.0<$ $|\eta|<5.8$, respectively. There is a sampling scintillation calorimeter surrounding the noble liquid calorimeters. The main concern for these systems is the radiation dose in the forward calorimeters, which is dominated by electromagnetic showers. The hadronic dose is distributed over a much larger volume. The maximum doses at standard luminosity to forward calorimeters expected in GEM are $400 \mathrm{Mrad} / \mathrm{SSCY}$ at the inner edge with $10 \mathrm{Mrad} / \mathrm{SSCY}$ of hadronic dose distributed uniformly in the $3<|\eta|<5.8$ region.

3) The muon system consists of several layers of cathode strip chambers arranged in three groups (superlayers) around the barrel and in the endcap. The barrel and endcap muon chambers cover $0.1<|\eta|<1.3$ and $1.4<|\eta|<2.46$, respectively. The outer radius of the muon system of about $9.5 \mathrm{~m}$ is restricted by a large superconducting magnet of total length $36 \mathrm{~m}$ that houses the entire detector and provides a field of 0.8 Tesla. The small angle coverage of the muon system is restricted by forward iron field shapers (FFS), which improve the forward muon momentum resolution by bending the solenoidal field to become normal to the muon direction. The main concern for the muon system is the random hits that occur through three separate processes: elastic scattering of neutrons from light elements, absorption of neutrons within the chamber and the interaction of the resultant photon in the gas of the chamber, and conversion of photons generated by neutrons in the material surrounding the muon chambers. The muon chambers tend to be far more sensitive to photons than to neutrons; in particular we estimate that in the barrel muon chambers more than $99.5 \%$ of the hits will be due to the processes involving a photon. We have simulated the effect of these random hits on the trigger and the pattern recognition capability of the muon chambers and concluded that the muon system can function with average neutron and photon fluxes less than about $10^{4} \mathrm{~cm}^{-2} \mathrm{~s}^{-1}[1,8]$. We have 
spent considerable effort in reducing random hits due to neutrons and photons in the muon chambers.

\subsection{COMPUTATIONAL METHODS}

We have performed this study in three stages: First, we identified the most important radiation sources by considering the deposited energy and the thickness of material between the source and the nearest sensitive detector. Second, we performed calculations to optimize the geometry and the shielding around the main sources. Finally, we performed detailed calculations of the particle fluxes in the entire detector and underground hall. We used the following simulation tools for these different tasks, and made consistency checks between the different simulations and simple calculations:

1) DTUJET93: This is a Monte Carlo program for hadronic multi-particle production in high energy $p p$ collisions. It is based on the two component dual parton model, which treats the single diffractive component, soft (low $P_{T}$, and hard (minijet and large $P_{T}$ ) processes in a unified and consistent way [9]. We used this model in all simulations to give us the distribution of particles from $40 \mathrm{TeV} p p$ interactions.

2) MARS12: This is a Monte Carlo program for simulations of hadronic and electromagnetic cascades and muons. The program emphasizes inclusive particle production and statistical weighting, and therefore, allows fast simulations. Versions of the code that interface with magnetic fields and accelerator transport exist, making this system well suited for accelerator shielding studies [10]. We used this code extensively for optimizing the shielding in the forward parts of the interaction region.

3) LAHET-MCNP: This Monte Carlo package [11] provides transport of hadrons through a combination of the FLUKA code and an extended version of the HETC code down to $1 \mathrm{MeV}$ for charged hadrons and $20 \mathrm{MeV}$ for neutrons. The MCNP code transports neutrons down to thermal energies. Electromagnetic processes are handled by ITS or EGS. We have not implemented the magnetic field options in LAHET for the studies described in this paper.

4) GEANT and CALOR: The main advantages of GEANT [12] are the ease of geometry specifications, and accurate tracking through magnetic fields. For this study we have used our implementation of GEANT containing a complete description of the GEM detector and the use of CALOR for low energy neutron transport $[13,14]$.

CALOR uses HETC with FLUKA87 for general hadronic transport, MORSE for neutrons with energy less than $20 \mathrm{MeV}$, and EGS for the transport of electromagnetic particles [15]. We used CALOR in two different ways: in combination with GEANT for 
low energy neutron transport and by itself for optimizing the shielding near the forward calorimeters.

\subsection{ENERGY LOSS CONSIDERATIONS}

There are three major sources of radiation: 1) particle production at the interaction point; 2) local beam loss in the collider tunnels; and 3) interactions of the $20 \mathrm{TeV}$ proton beams with the residual gas in the beam pipe. The relative contributions of each of these sources to the total particle flux and the deposited energy will depend on the luminosity and the beam current. At the standard SSC luminosity of $10^{33} \mathrm{~cm}^{-2} \mathrm{~s}^{-1}\left(10^{8}\right.$ interactions $\left./ \mathrm{s}\right)$ and beam current of $1.3 \times 10^{14}$ protons in each ring the radiation levels will be dominated by the particle production at the interaction point.

\subsection{COLLISIONS AT THE INTERACTION POINT}

Figure 2 shows the fraction of the total energy from the interaction point emitted as a function of pseudorapidity. DTUJET93 produces a pseudorapidity $(\eta)$ plateau of 7.5 charged particles per unit pseudorapidity (for $-5<\eta<5$ ) and the mean transverse momentum of $0.6 \mathrm{GeV}$ in agreement with extrapolation of data from lower energies. There is no data in the far forward regions $(|\eta|>6.0)$; therefore we assign an uncertainty of a factor of 2 to the differential distribution of particles in the forward regions. Though the uncertainty in the distribution of radiated energy should be smaller, we conservatively assign an error of a factor 2 to the energy intercepted by the various forward elements [9]. For a detector covering $|\eta|<6$, we see that roughly $5 \%$ of the energy is deposited in $3<|\eta|<6$ and more than $90 \%$ of the energy is beyond $|\eta|-6$. At least part of this energy must be intercepted in a thick collimator placed in front of the low beta quadrupoles to reduce the heat load on the cryogenic magnets and the radiation damage to the coil insulation. The energy intercepted by the forward calorimeters (5\%) is less than the energy into to the collimators (about 30\%), but due to the proximity of sensitive muon detectors to the forward calorimeters, both the collimator and the forward calorimeter regions will be the dominant sources of background neutron and photon fluxes.

\subsection{LOCAL BEAM LOSS}

Beam loss in the walls of the beam pipe occurs continuously around the accelerator. The maximum of the accelerator beta function occurs approximately 70 meters from the interaction point [16], where the beam loss is particularly severe. Using the MARS12 code system [17] we have estimated neutron and charged particle fluxes due to this beam loss by making simulations of proton orbits around the accelerator and of the hadronic 
cascades due to the lost protons. For the interaction region geometry anticipated for GEM, we find that the contribution from this source is negligible.

\subsection{BEAM-GAS INTERACTIONS}

The particle fluxes due to interactions of $20 \mathrm{TeV}$ beam protons with atoms of the residual gas (beam gas interactions) in the evacuated beam pipe was computed using the MARS12 code system. The calculation assumed a pressure of $10^{-8}$ torr of nitrogen in the room temperature regions of the beam pipe (which extends up to the low beta quadrupoles located $35 \mathrm{~m}$ from the interaction point). A density of $3.6 \times 10^{8} \mathrm{~mol} / \mathrm{cm}^{3}$ $\left(80 \% \mathrm{H}_{2}\right.$ and $\left.20 \% \mathrm{CO}, \mathrm{CO}_{2}\right)$ was assumed for the cold regions of the beam pipe inside the magnets. This density corresponds to a loss of $5 \times 10^{3} \mathrm{protons} / \mathrm{m} / \mathrm{s}$ due to nuclear interactions and Coulomb scattering from each of the two rings in the 100-m-long experimental hall. Taking into account the tails from other interaction regions and scrapers, RF noise, and collective beam-beam interactions, one arrives at the number of $8.3 \times 10^{3}$ protons $/ \mathrm{m} / \mathrm{s}$ from both rings. The total then comes out to be $1.8 \times 10^{4}$ protons $/ \mathrm{m} / \mathrm{s}$; we have used the value of $2 \times 10^{4} \mathrm{protons} / \mathrm{m} / \mathrm{s}$ [18]. Beam gas interactions as far as 120 meters away from the interaction point contribute to the particle fluxes in the central cavity of the detector. For GEM, we have estimated that at the standard SSC luminosity the contribution to the neutron flux due to beam gas interactions is less than 3\% everywhere in the detector.

The amount of energy lost from the collider at the interaction point $\left(4.0 \times 10^{9} \mathrm{TeV} / \mathrm{s}\right)$ is much greater than the beam loss in the accelerator magnets near the interaction point (approximately $4.0 \times 10^{7} \mathrm{TeV} / \mathrm{s}$ ) or the beam loss in the residual gas of the vacuum pipe $\left(4.0 \times 10^{7} \mathrm{TeV} / \mathrm{s}\right)$. The energy consideration alone is sufficient to assert that the interation point is the major source of baskground radiation in the SSC.

\subsection{SHIELDING DESIGN AND OPTIMIZATION}

We have used the above mentioned computer simulations to design different components of the shielding (Figure 1) and optimize it for cost, weight, and access to the detector. The most difficult aspect of this optimization was hit rates in the muon chambers discussed earlier. We have concentrated on removing neutrons as close to their sources (the collimator and the forward calorimeter) as possible to reduce the muon chamber rates. We have accomplished our task by hermetically sealing the entire beamline: The thick calorimeter serves as a shield in the central region, and the concrete and the field shaper protect the muon system from radiation at the beam pipe, the 
collimator, and the quadrupoles. Below, we briefly describe each of the major simulation studies; the details can be found in Reference [2].

\subsection{LOCATION AND APERTURE OF THE COLLIMATORS}

The iron collimators protect the cryogenic magnets from the intense radiation from the interaction point. The energy going into the magnets is, however, a weak function of the solid angle of the collimator hole because the distribution of energy from the interaction point is extremely forward-directed (Figure 2). We have also found that the neutron and photon flux in the detector from hadronic showers in the collimators is insensitive to the collimator location. This is because some fraction of the radiation from the collimator undergoes secondary reactions in the concrete walls of the collision hall and the forward regions of the detector, reducing the effect of the collimator as a localized source. Therefore, the location of the collimator and first quadrupole is largely determined by considerations of luminosity, space needed for shielding, and detector access. For GEM, we have chosen a $3 \mathrm{~m}$ (16.7 interaction lengths) deep iron collimator with a $25 \mathrm{~mm}$ diameter inner aperture placed $32 \mathrm{~m}$ from the interaction point.

\subsection{SHIELDING FOR THE COLLIMATORS}

We expect that the best shields will be composites made of heavy metals to contain the high energy cascades in a small volume, and light materials such as concrete or polyethylene to moderate and absorb the low energy neutron leakage. The iron of the collimator serves as the heavy core that contains the high energy shower. The largest reduction of neutron fluxes in the detector volume results from the addition of the albedo trap, which is the thick layer of concrete in front of the collimator surrounding the beam

pipe in Figure 1. We have found the neutron flux attenuation largely insensitive to the geometry of the concrete around the collimator when at least $5 \mathrm{~m}$ of concrete is present to trap the albedo. We also find that the use of special concretes such as barite [19] with increased barium and boron contents leads to shielding designs with smaller volumes. We calculate that the configuration of shielding shown in Figure 1 gives two orders of magnitude reduction in neutron fluxes on the boundary. A factor of 2 reduction results in the endcap muon system because there both the forward calorimeter and the collimator contribute equally. We have chosen to extend the collimator shield up to the iron field shaper to eliminate gaps and trap majority of the albedo.

\subsection{THE FORWARD CALORIMETER}

The location of this shielding in the middle of the sensitive muon system, and the function of the forward calorimeter as an active detector, present special constraints on 
this shielding design. First, we must place sufficient high density material at the location of the forward hadronic calorimeter transverse to the beam axis to attenuate all charged particles. Second, we cannot place neutron absorbing material such as borated polyethylene in proximity to the muon chambers, which are sensitive to the photons emitted as a result of neutron absorption on boron (the reaction $\mathrm{B}^{10}(\mathrm{n}, \alpha) \mathrm{Li}^{7}$ and subsequent decay of $\mathrm{Li}^{7}$ through photon emission $0.477 \mathrm{MeV}$ ). In order to quantify the number of neutrons injected into the muon system by particle interactions, we have performed Monte Carlo simulations that include the endcap calorimeter, the forward calorimeter, and the iron field shaper [2,20]. We find that the majority of the neutrons are produced by particles striking the inner edge of the forward calorimeter $(5.6<|\eta|<$ 5.8). They leak into the muon system either through gaps in the endcap and forward calorimeter or through the large gap between the detector and the iron field shaper. We can reduce the leakage by placing passive copper shielding around the forward calorimeter and concrete between the forward calorimeter and the field shaper as shown in Figure 1(b). In particular, we have calculated that rates in the inner most endcap muon chambers can be reduced by at least $60 \%$ by using a $\mathrm{Cu}$-Borated polyethylene-Cu sandwich with optimized thicknesses for each layer. Alternatively, shaping the inner edge to be projective can eliminate this source entirely; practical considerations of detector resolution, support structures, and ease of fabrication made this alternative unsuitable in our case.

\subsection{BEAM PIPE AND FIELD SHAPER CONSIDERATIONS}

GEM has chosen a compact forward calorimeter that fits in the endcap calorimeter without leaving large gaps; it provides a natural, effective shield for the muon system. If the forward calorimeter were absent, the field shaper would become the source of radiation. Though the choice for the forward calorimeter has made the design of the detector very challenging because of the large radiation dose and limited access, the design of the shielding is simplified. It is natural to design the other beam line elements so that they are entirely in the shadow of the forward calorimeter. Thus, We have designed a flared beam pipe and a field shaper with a wide hole $(120 \mathrm{~cm}$ diameter $)$ between the back end of the forward calorimeter and the collimator, so that all material is in the shadow of the forward calorimeter. Particles from the interaction point passing through the hole in the forward calorimeter cannot interact until they enter the iron collimator in front of the low beta quads. Secondary products from the showers initiated at the inner edge of the forward calorimeter are the only significant source of interactions in this section of the beam pipe. The flaring of the beam pipe reduces the number of 
hadronic interactions by more than a factor of 10 , with a corresponding decrease in the neutron flux near the beam pipe between the forward calorimeter and the collimator.

\subsection{CENTRAL DETECTOR CAVITY}

The albedo neutron and photon fluxes in the central cavity are affected by the volume of the cavity, the composition of the electromagnetic calorimeter, and by the shielding material placed in front of the calorimeter. Albedo neutrons from the forward calorimeter must travel a long narrow path through the endcap; majority of them are absorbed before reaching the central cavity. Therefore, the radiation environment of the central cavity is not affected significantly by changes in the forward calorimeter or the other forward elements. As shown in Reference [3], the neutron flux within a spherical cavity is mostly uniform and scales as $1 / \mathrm{r}^{2}$, where $r$ is the radius of the cavity; for the cylindrical cavity of GEM with characteristic dimensions of radius and length the distribution of flux will deviate by a small amount from this idealized behavior. Furthermore, the flux is proportional to $(1+a)$, where $a$ is the mean number of reflections that a neutron experiences before being absorbed. Borated polyethylene placed on the walls of the central detector cavity should reduce the neutron flux by fast absorption. However, photons emitted as a result of neutron absorption on boron can increase the photon flux in the central detector cavity.

We have performed calculations of neutron and photon fluxes for different amounts of borated polyethylene to understand the relative sizes of these effects. The neutron flux increases by a factor of approximately 1.5 in the forward regions near the endcap calorimeter due to the increased flux and energy of the primary particles striking this

region. The borated polyethylene reduces the flux by a factor of 2 to 5 , depending on location. Increasing the borated polyethylene thickness does not always produce a proportionate reduction in the flux. We believe that this is so because the polyethylene has a smaller effect on the high energy component of the albedo, and also because the polyethylene itself starts contributing to the albedo. The shielding configuration that we have chosen is shown in Figure 1b. We have designed the polyethylene in front of the endcap calorimeter to be thinner at larger angles so that it has as little effect on the overall electromagnetic resolution as possible.

\subsection{RESULTS AND CONCLUSIONS}

Figure 3 is a schematic of the detector model used in our simulations. It can be overlaid on Figures 4-7 to obtain the neutron or gamma fluxes in various regions of the detector. Contour plots of the neutron flux, produced by the GEANT-CALOR package, 
for the unshielded and shielded detector are shown in Figures 4. Figures 5 contain the corresponding gamma flux. Results of our LAHET-MCNP calculation for the unshielded and shielded detector are in Figures 6 and 7 for neutrons and photons, respectively. Neutrons of all energies and photons above $10 \mathrm{keV}$ ( $1 \mathrm{keV}$ for LAHET-MCNP) are included in these figures. Both of these simulations contained a description of the detector and the hall that was detailed and asymmetric around the beam axis. In the interest of clarity we have presented the results for one quadrant of the detector as if the geometry was symmetric.

There are several differences in the two major simulations: though the detector geometry was similar in both simulations, the magnetic field in both the detector and the low beta quadrupoles was absent from the LAHET-MCNP simulation. The magnetic field focuses low $P_{T}$ charged particles down the beam pipe and causes the energy from the interaction point to be deposited over a longer distance. Reference [3] shows that this effect should reduce the neutron flux by about 30 to $40 \%$ in the muon system for the GEM detector parameters. Thus a large part of the difference between the two simulations in the muon system can be attributed to the magnetic field. We have found that in the central cavity the GEANT-CALOR calculation gives neutron fluxes that are approximately a factor of 3 lower than the LAHET-MCNP calculation. The neutron spectrum in the central cavity from GEANT-CALOR is very similar but has a significant thermal component. This could be resulting from small differences in material descriptions or the cross sections used [21] and the transport algorithms. Lastly, the GEANT-CALOR simulated photon flux includes contributions from both high energy electromagnetic showers and $(n, \gamma)$ interactions. The LAHET-MCNP simulation of photons includes only the $(n, \gamma)$ induced photons. Of course, this difference is not significant in the muon system.

The shielding reduces the neutron flux in most of the muon system to the range of 1-5 $\times 10^{3} \mathrm{~cm}^{-2} \mathrm{~s}^{-1}$ at the standard luminosity of $10^{33} \mathrm{~cm}^{-2} \mathrm{~s}^{-1}$. The photon flux in the muon system above $0.1 \mathrm{MeV}$ is approximately $30 \%$ of the neutron flux in both simulations. The muon chambers surrounding the forward hadronic calorimeter are expected to receive neutron and photon fluxes that are approximately twice as large. The average neutron flux in the central cavity is calculated by LAHET-MCNP to be $4 \times 10^{5} \mathrm{~cm}^{-2} \mathrm{~s}^{-1}$; approximately half of these neutrons will be above $100 \mathrm{keV}$ where the silicon displacement cross section increases abruptly by an order of magnitude. The photon flux above $0.1 \mathrm{MeV}$ in the cavity is approximately twice the neutron flux if contribution from 
albedo photons from electromagnetic showers is included. The photon flux from $(n, \gamma)$ interactions is approximately the same as the neutron flux.

\subsection{SPECTRUM}

Figures 8 and 9 show the spectra of neutrons and photons in the barrel muon system and the central cavity of the shielded detector, respectively. These spectra were obtained as fluxes across cylindrical surfaces located in the muon barrel and the central cavity.

The spectrum of neutrons depends on the details of the shielding and the location in the detector. The thermal part of the neutron spectrum poses special problems since it is highly dependent on the water content of the air and concrete in the hall. It also depends on the presence of isotopes with very high thermal neutron absorption cross-sections; these may be present in a real experimental situation and absent in the simulation described here. We find that although our two major simulation agree on the shape of nonthermal part of the spectrum, they disagree on the height of the thermal peak. We conclude that between 10 to $30 \%$ of the neutrons are thermal in the muon system of the shielded detector. The neutron spectrum in the central cavity peaks at kinetic energies of a few $\mathrm{MeV}$. It has a high energy component resulting from the direct production of neutrons in the $p p$ interactions. Except for a significant thermal component in the GEANT-CALOR prediction, both simulations predict a very similar neutron spectrum.

The spectrum of photons contains many peaks due to $(n, \gamma)$ reactions on boron $(0.48$ $\mathrm{MeV}$ ), hydrogen (2.2 MeV), and other elements (collection of peaks near $8 \mathrm{MeV}$ ). LAHET-MCNP predicts a higher number of lower energy photons in muon system, especially within the $0.511 \mathrm{MeV}$ peak due to positron annihilation. The $0.511 \mathrm{MeV}$ photons result from pair production interactions of the higher energy photons. Although this process is present in GEANT-CALOR, we are uncertain about the origin of this discrepancy.

\subsection{CONCLUSION}

The uncertainties in this calculation result from four sources: 1) the particle production model, 2) knowledge and implementation of the various cross sections in the materials of the detector, 3) inexact modeling of the detector geometry and materials for computational convenience, 4) absence of the magnetic field in the LAHET-MCNP simulation. We assign an error of a factor of 2 for the first uncertainty. The other two uncertainties can be estimated by comparing the different Monte Carlo codes, which use different cross section libraries and tracking algorithms. We find agreement within a factor of 2 between the results of several codes used in this study. Although part of the 
disagreement can be attributed to the magnetic field as discussed earlier, we have conservatively chosen to consider the difference between the simulations as representative of the difference between simulation and the real experiment. Therefore adding the two uncertainties in quadrature, we are confident that our estimates are accurate within an overall factor of 3 . The contribution to the error from Monte Carlo statistics is small on this scale.

We have demonstrated detailed simulations of the soft neutron and photon radiation in a major SSC detector using different Monte Carlo programs. We have also shown our methods for optimizing the design of the shielding. Our current results have been used to compute occupancy levels and survival limits in the GEM detector. As a result of these studies, we have developed a shielding and collimator configuration, and an overall layout of the experiment that will ensure that the detector will operate with its design performance for at least 10 years at the standard luminosity of $10^{33} \mathrm{~cm}^{-2} \mathrm{~s}^{-1}$. We have also demonstrated that the muon system can work at the high luminosity of $10^{34} \mathrm{~cm}^{-2} \mathrm{~s}^{-1}$ without difficulty with random hit rates.

This study required close co-ordination amongst many members of the GEM collaboration. We would especially like to acknowledge contributions of Gerry Chapman, Joe Coyne, Vladimir Gavrilov, Louisa Hansen, Roger McNeil, Kate Morgan, Harvey Newman, Barry Barish, and Bill Willis. This work was supported by the U.S. Department of Energy under contract No. DE-AC35-89ER40486 and No. DE-AC02$76 \mathrm{CH} 00016$.

\section{REFERENCES}

[1] "The GEM Technical Design Report," by the GEM collaboration, SSC-SR-1219, April 30, 1993.

[2] "Radiation Environment and Shielding for the GEM detector at the SSC," M. Diwan et al., SSCL-SR-1223. July 1993.

[3] "Radiation Levels in the SSC Interaction Regions," Edited by D. E. Groom, SSCSR-1033, June 10, 1988. See also "Radiological Safety Aspects of the Operation of Proton Accelerators," R.H. Thomas and G.R. Stevenson, IAEA technical reports series no. $283,1988$. 
[4] "A Monte Carlo Calculation of the Neutron Flux in the L* Detector," D.M. Lee, W.W. Kinnison, and W.B. Wilson, The Conference Record of the IEEE1990 Nuclear Science Symposium, Arlington, Virginia, October 22-27,1990, Vol.2,P. 887

[5] "Calculation of the Production and Decay of Radionuclides in the Hadron Calorimeter of the L* Detector for the SSC, "W.B. Wilson, T.R. England, W.W. Kinnison, and D.M. Lee, Los Alamos National Laboratory document LA-UR-91-999 (March 1991).

[6] "Radiation Damage Considerations at a High Luminosity Collider -The Interaction Region," D.M. Lee, SUPERCOLLIDERS and SUPERDETECTORS Extendingthe Frontiers of Energy and Luminosity, William A. Barletta and Heinrich Leutz, editors, Ettore Majorana Centre for Scientific Culture, Erice, Sicily 1992

[7] "Radiation Resistance of Semiconductor Detectors and Associated Electronics," G. Hall, CERN-90-10, Large Hadron Collider Workshop, Aachen Oct. 1990. page 693.

[8] "Hit Rate estimation for the GEM Central Tracker and Muon Systems," Yuri Fisyak, GEM-TN-93-390, June 1993.

[9] J. Ranft, et al., Multiparticle Dynamics, Seewinkel, Austria, 1986. J. Ranft, Presentation in the Simulating Accelerator Radiation Environments Workshop, Sante Fe, New Mexico, Jan. 1993, to be published. See also J. Ranft, et al., UL-92-7 and UL-HEP93-01, Leipzig, Germany. We have used the February 1, 1993 version of DTUJET.

[10] "MARS12 code system," N. Mokhov, Presentation in the Simulating Accelerator Radiation Environments Workshop, Santa Fe, New Mexico, Jan. 1993, to be published. Also see N. Mokhov, MARS10 Code Manual, FERMILAB-FN-509, 1989.

[11] "User Guide to LCS: the LAHET Code System," Richard E. Prael and Henry Lichtenstein, Los Alamos National Laboratory, LA-UR-89-3014, Sep. 1989. "MCNP: A General Monte Carlo Code for Neutron and Photon Transport," Judith F. Briesmeister, LA-7396-M Rev. 2, Sep. 1986. "Radiation Calculations using LAHET, MCNP, CINDER90, " Proceedings of the II International Conference on Calorimetry in High Energy Physics, Corpus Christi, LA-UR-89-3014, Oct. 1992

[12] "Hadronic Cascade Simulation in GEANT," K. Lassila, CERN-CN-91-13, Dec. 1991. "GEANT: Simulation Program for Particle Physics Experiments. User's Guide and and Reference Manual," R. Brun, R. Hagelberg, M. Hansroul, J.C. Lassalle, CERN$\mathrm{DD} / 78 / 2$ Rev. July 1978. 
[13] "The GEANT-CALOR Interface," C. Zeitnitz, T.A. Gabriel, International Conference on Monte Carlo Simulation in High Energy and Nuclear Physics, Proceedings (to be published), Tallahassee, 1993. See also J.O. Johnson, T.A. Gabriel, "Development and Evaluation of a Monte Carlo Code system for analysis of Ionization Chamber Responses," ORNL/TM-10196, July 1987.

[14] "CALOR: A Monte Carlo Program Package for the Design and Analysis of Calorimeter Systems," T.A. Gabriel, J.D. Amburgey, B.L. Bishop, ORNL/TM-5619, Apr. 1977. "User's Guide for the FLUNEV Code," J.M. Zazula, Desy-internal-rep D3-90-66, Jan. 1990. "FLUKA and KASPRO Hadronic Cascade Codes," J. Ranft, Erice 1978, Proceedings, Computer Techniques in Radiation Transport and Dosimetry.

[15] "The Physics of Compensating Calorimetry and the New CALOR89 Code System," T.A. Gabriel, J.K. Amburgey and B.L. Bishop, IEEE Trans. Nucl. Sci. 36 (1989) p14. See also "Applications Guide to the MORSE Monte Carlo Code," S.N. Cramer, ORNL/TM-9355(1985). "The EGS4 Code System," W.R. Nelson, H. Hirayama, D.W.O. Rogers, SLAC-265 (1985).

[16] Y. Nosochkov, E. Courant, A. Garren, D. Ritson, T. Sen, R. Stiening, SSCL-368, 1993.

[17] I. Baishev, A. Druzhdin, and N. Mokhov, SSC-306, 1991.

[18] N. Mokhov, Collider ARC PDRR, May 26-27, 1993.

[19] "Concrete Radiation Shielding: Nuclear Physics, Concrete Properties, Design and Construction," Wiley, New York, 1989. The concrete that we propose to use has a density of $3.2 \mathrm{gm} / \mathrm{cm}^{3}$; it contains, by weight, $40 \%$ barium, $35 \%$ oxygen, $9.6 \%$ sulfur, $8.5 \%$ calcium, $1.8 \%$ silicon, $1 \%$ iron, $1 \%$ boron, $0.85 \%$ hydrogen, and the rest are trace minerals.

[20] "Studies on the Reduction of Neutron and Gamma Leakage from the Forward Region of the GEM detector," B. R. Moore, GEM-TN-93-429, July 1993.

[21] "Neutron Cross Sections," D. I. Garber, R. R. Kinsey, BNL-325, Brookhaven National Laboratory, January 1976. For the Los Alamos cross sections library see "A Nuclear Cross Section Data Handbook," Harl'o M. Fisher, LA-11711-M-Manual, Los Alamos National Laboratory, Dec. 1989. 


\section{FIGURES}

Figure 1. Detector and shielding configuration used in this study to calculate particle fluxes and dose rates. Same calculations were also performed without the indicated shielding. a) Elevation view of half of the detector and shielding. b) Details of the shielding near the calorimeters.

Figure 2. Fraction of the total energy from the interaction point emitted as a function of pseudorapidity.

Figure 3. Schematic of one quadrant of the detector model used in our simulations. This can be overlaid on Figures 4 to 7 .

Figure 4. Neutron flux, in units of $10^{\mathrm{n}} \mathrm{cm}^{-2} \mathrm{~s}^{-1}$, where the contour indicates the power $\mathrm{n}$, in the unshielded and shielded detector (calculated using the GEANT-CALOR package). Neutrons of all energies are included in the plot.

Figure 5. Photon flux, in units of $10^{\mathrm{n}} \mathrm{cm}^{-2} \mathrm{~s}^{-1}$, where the contour indicates the power $\mathrm{n}$, in the unshielded and shielded detector (calculated using the GEANT-CALOR package). Photons have an energy cutoff $E>10 \mathrm{keV}$.

Figure 6. Neutron flux, in units of $10^{n} \mathrm{~cm}^{-2} \mathrm{~s}^{-1}$, where the contour indicates the power $\mathrm{n}$, in the unshielded and shielded detector (calculated using the LAHET-MCNP package). Neutrons above $20 \mathrm{MeV}$ are not included in the plot. They represent a negligible contribution to the flux away from the beam-pipe.

Figure 7. Photon flux, in units of $10^{n} \mathrm{~cm}^{-2} \mathrm{~s}^{-1}$, where the contour indicates the power $\mathrm{n}$, in the unshielded and shielded detector (calculated using the LAHET-MCNP package). Photons have an energy cutoff $E>1 \mathrm{keV}$, however as Figures 8 and 9 show there are very few photons below $10 \mathrm{keV}$.

Figure 8. Neutrons ( $n$ ) and photons $(\gamma)$ energy spectra in the barrel muon system of the shielded detector calculated using (a) the GEANT-CALOR package and (b) the LAHETMCNP package. The normalization is arbitrary in these plots. The peaks at the higher end of the neutron spectrum are due to particular resonances in iron. The peak at 2.2 $\mathrm{MeV}$ in the photon spectrum are due to $(\mathrm{n}, \gamma)$ reactions on hydrogen. The peak around 8 $\mathrm{MeV}$ in the photon spectrum is actually a collection of peaks within the same bins. The two simulations differ with respect to the fraction of thermal neutrons and the lower energy photons (especially at $0.511 \mathrm{MeV}$ ), some of which are produced by interactions of the higher energy photons. 
Figure 9. Neutron (n) and photon ( $\gamma$ ) energy spectra in the central detector cavity with the polyethylene shielding using the LAHET-MCNP package. These spectra were obtained at a radius of $10 \mathrm{~cm}$. The neutron spectrum includes high energy neutrons produced in the $p p$ collisions. The photon spectrum includes only photons from $(n, \gamma)$ reactions. The peaks at $0.48 \mathrm{MeV}$ and $2.2 \mathrm{MeV}$ are from captures on boron and hydrogen, respectively. The width of the peaks is mainly due to binning. The GEANT-CALOR spectra are similar, except that the neutron spectrum is predicted to have a 10 to $20 \%$ thermal component. 
a)

$50 \mathrm{~m}$

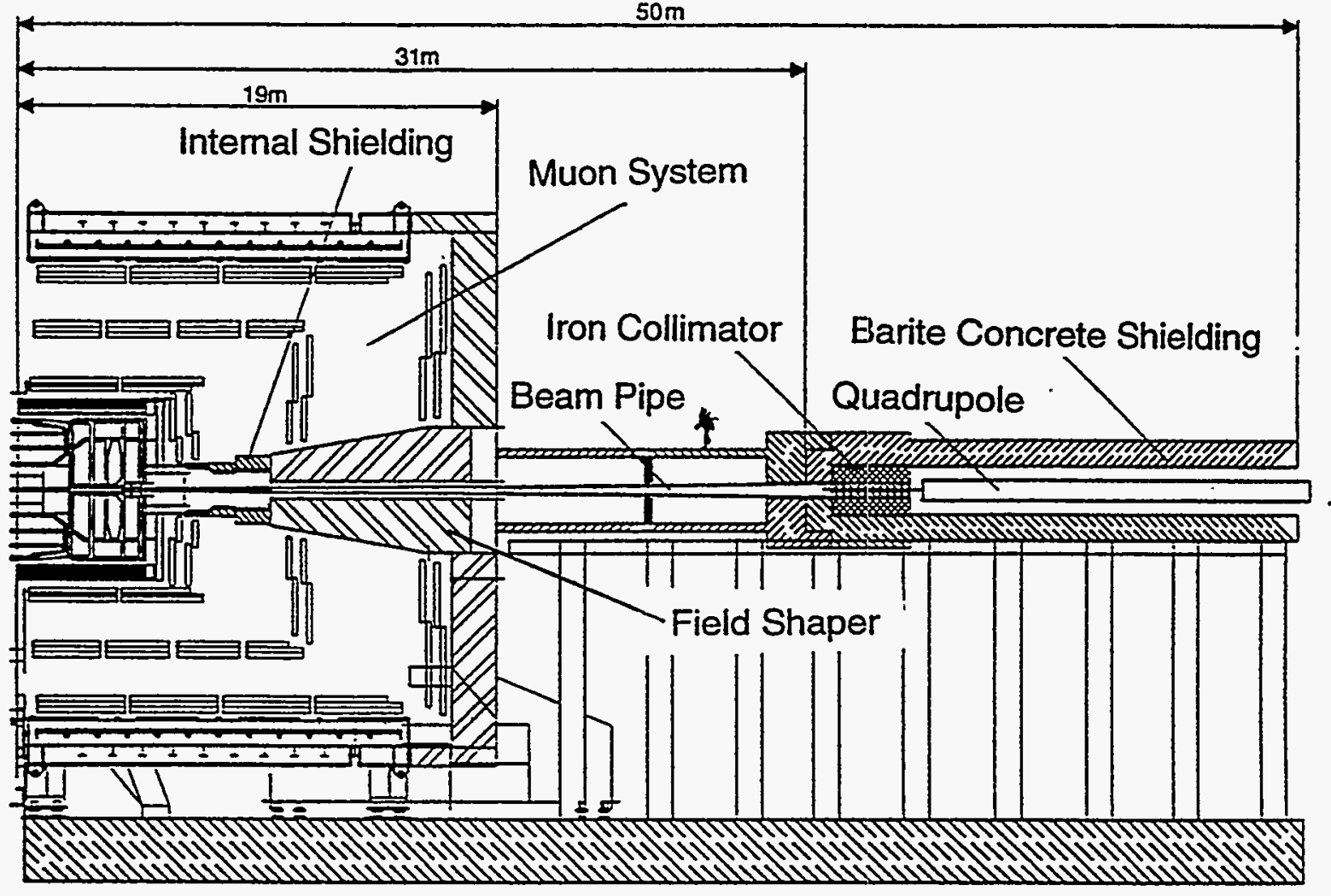

b)

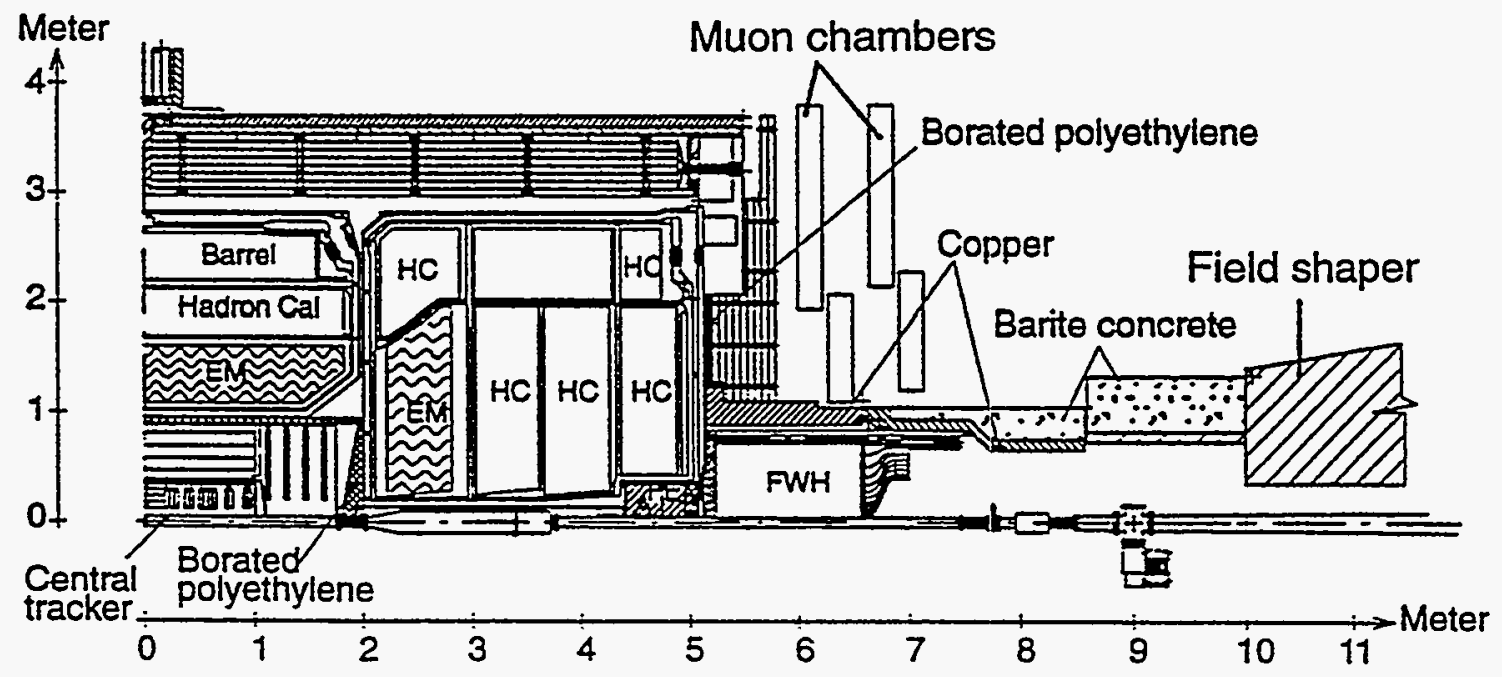

Figure 1 


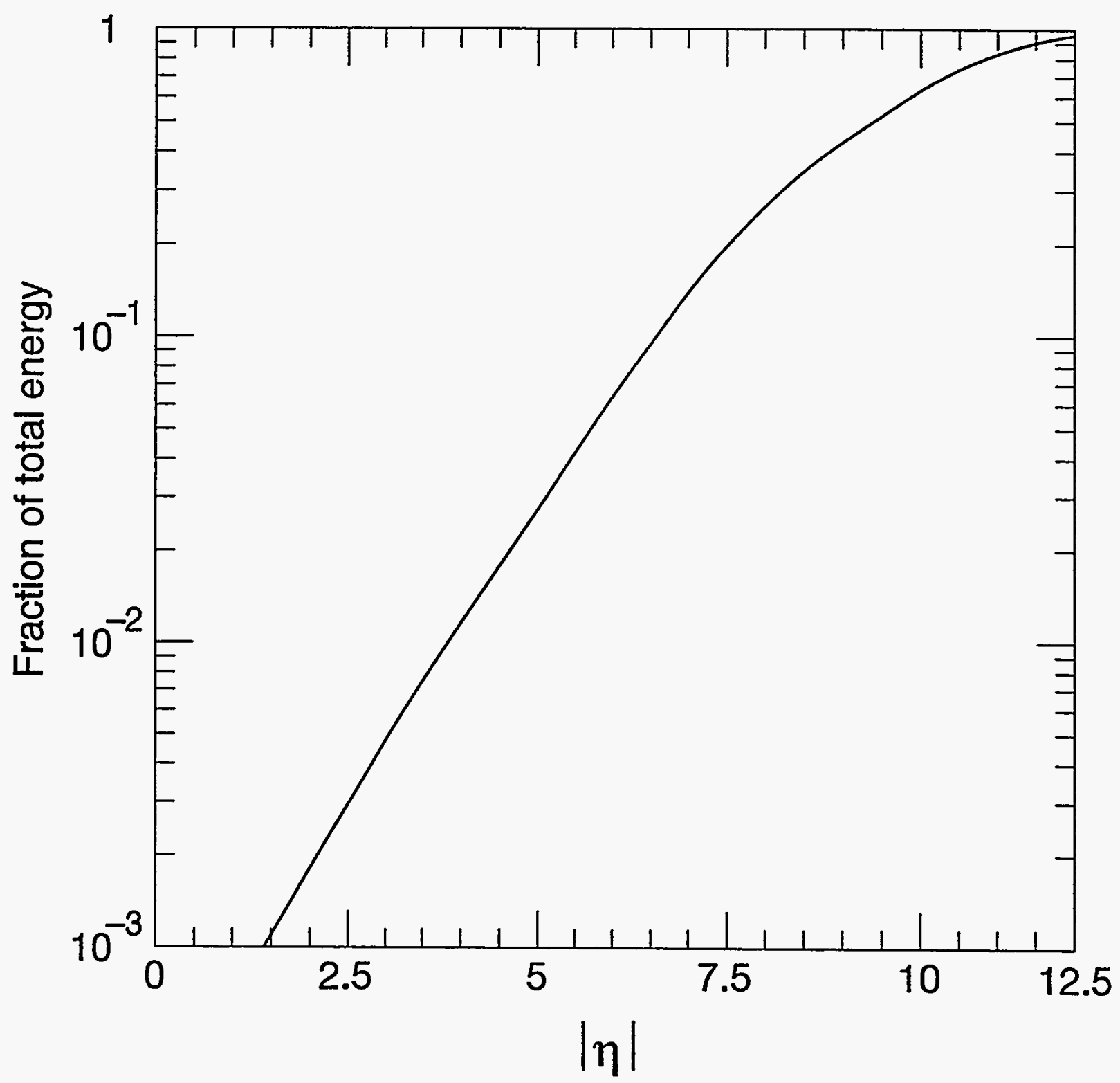

Figure 2 

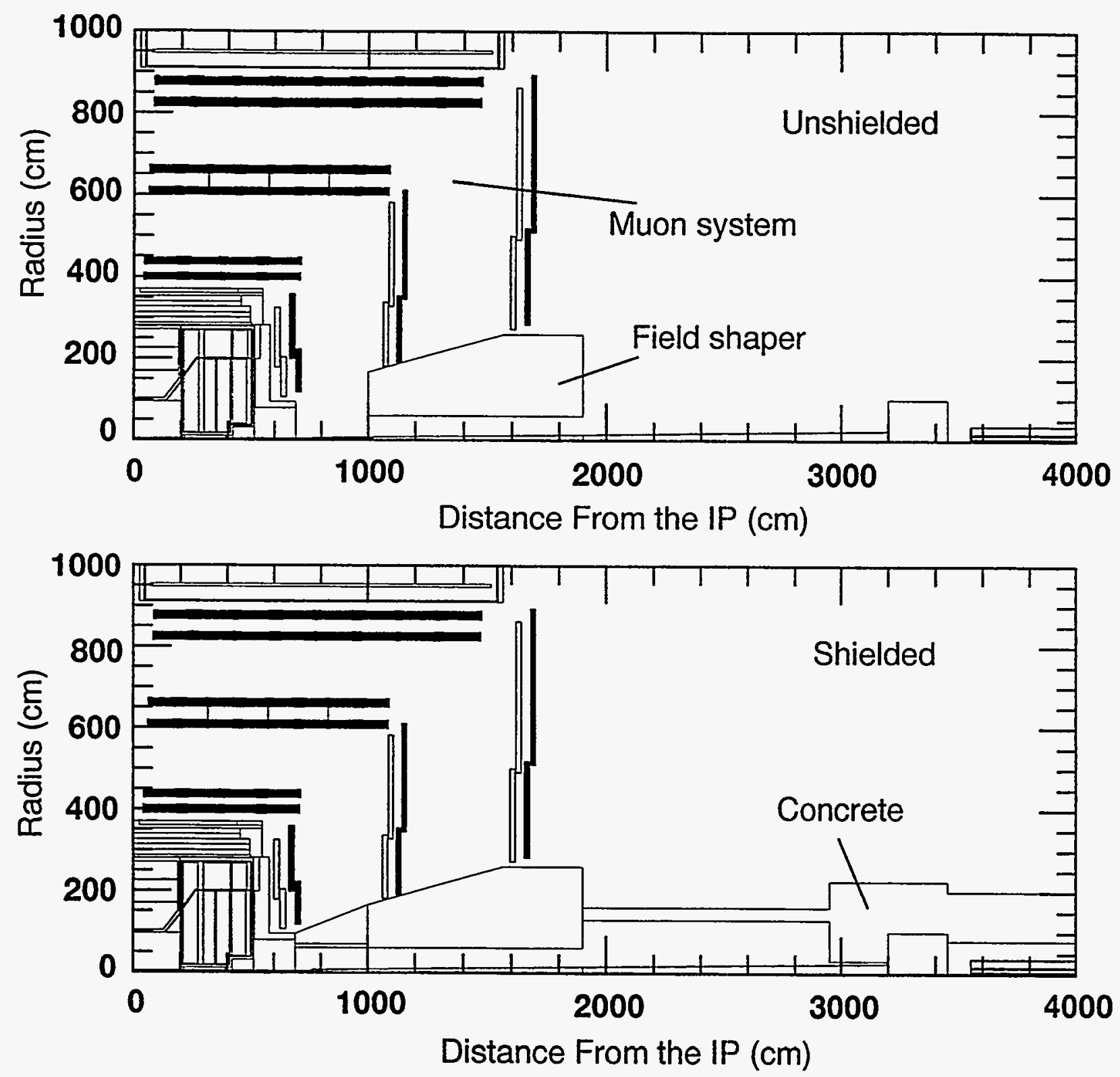

Figure 3 

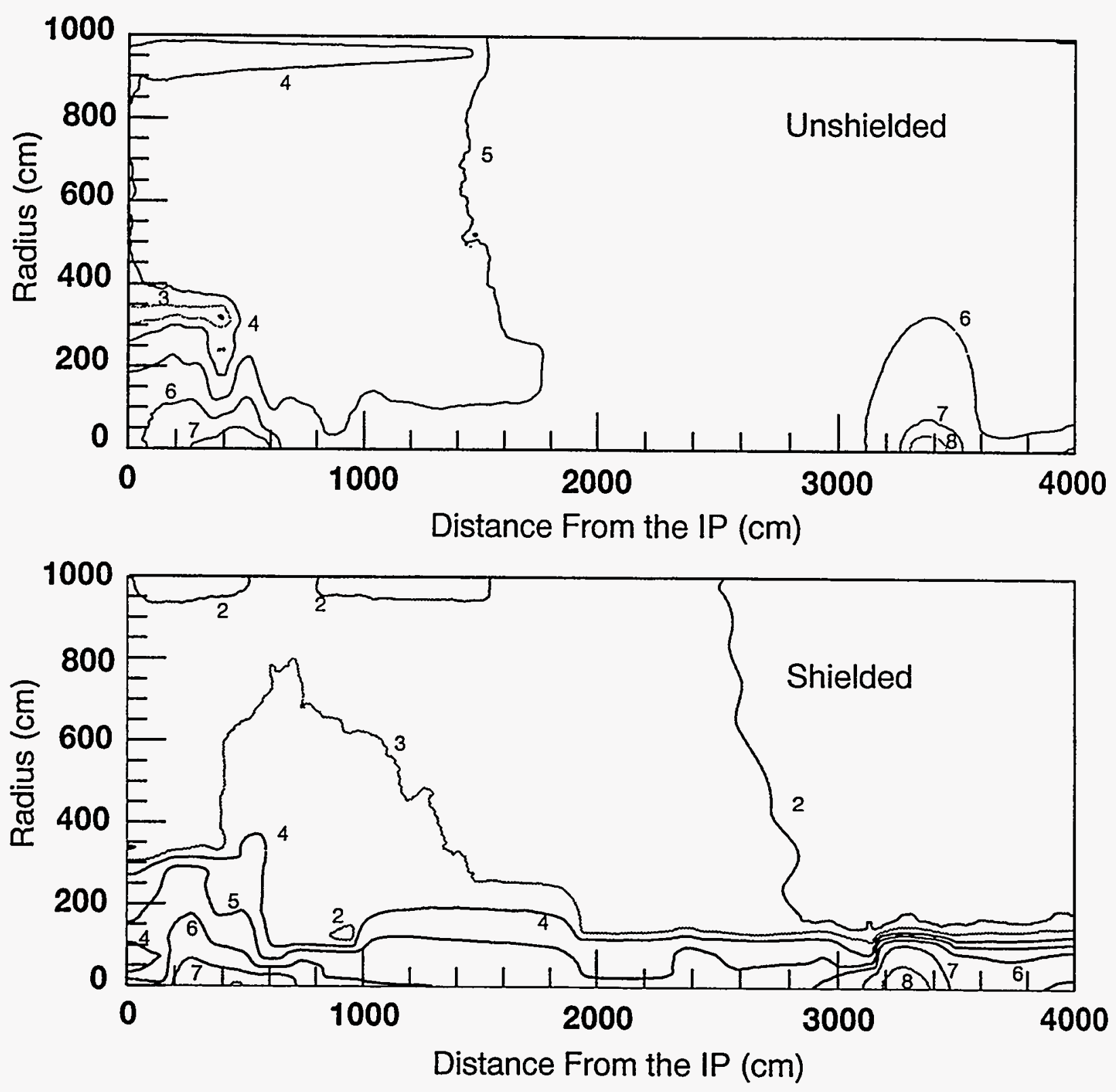

Figure 4 

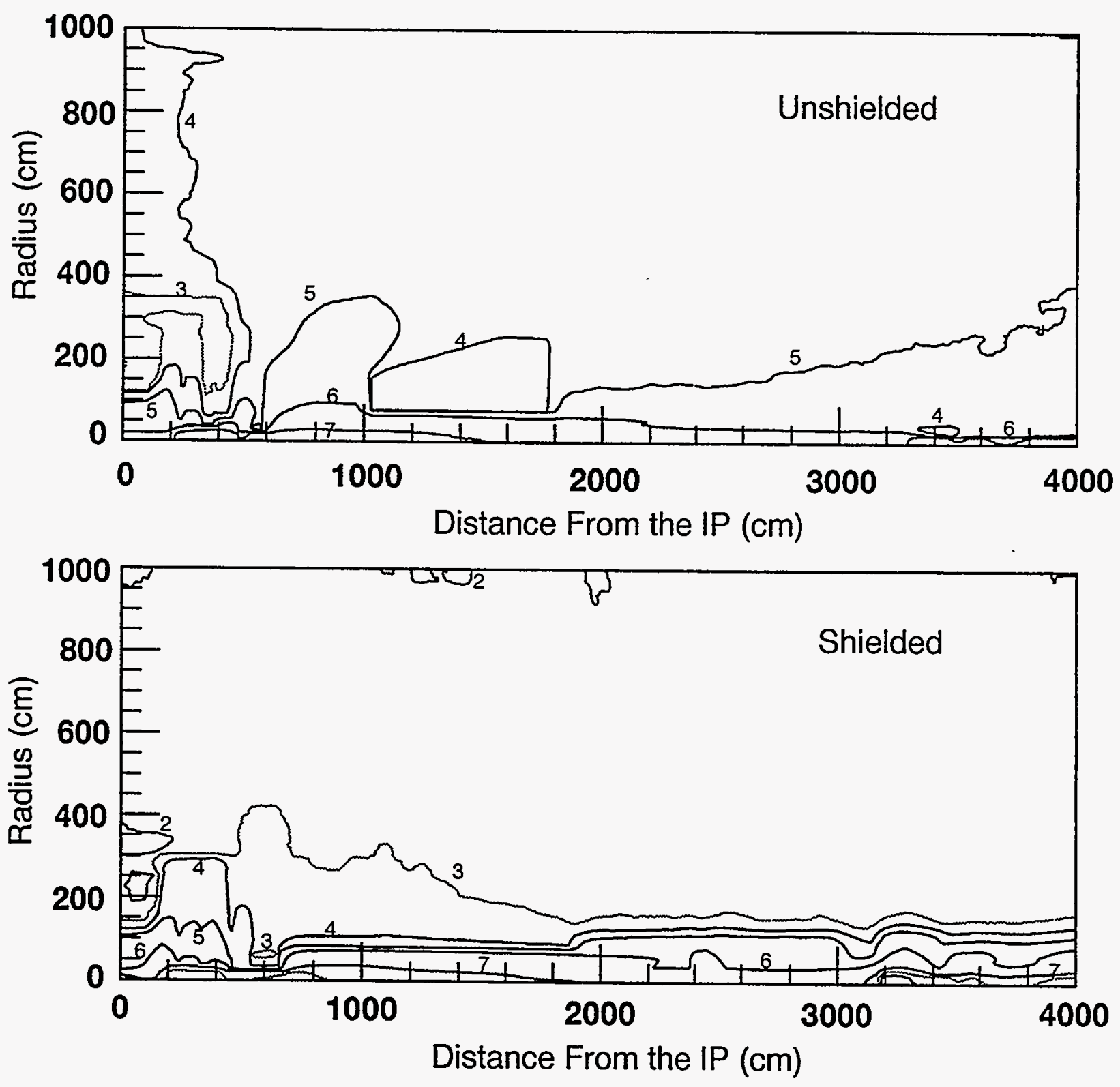

Figure 5 

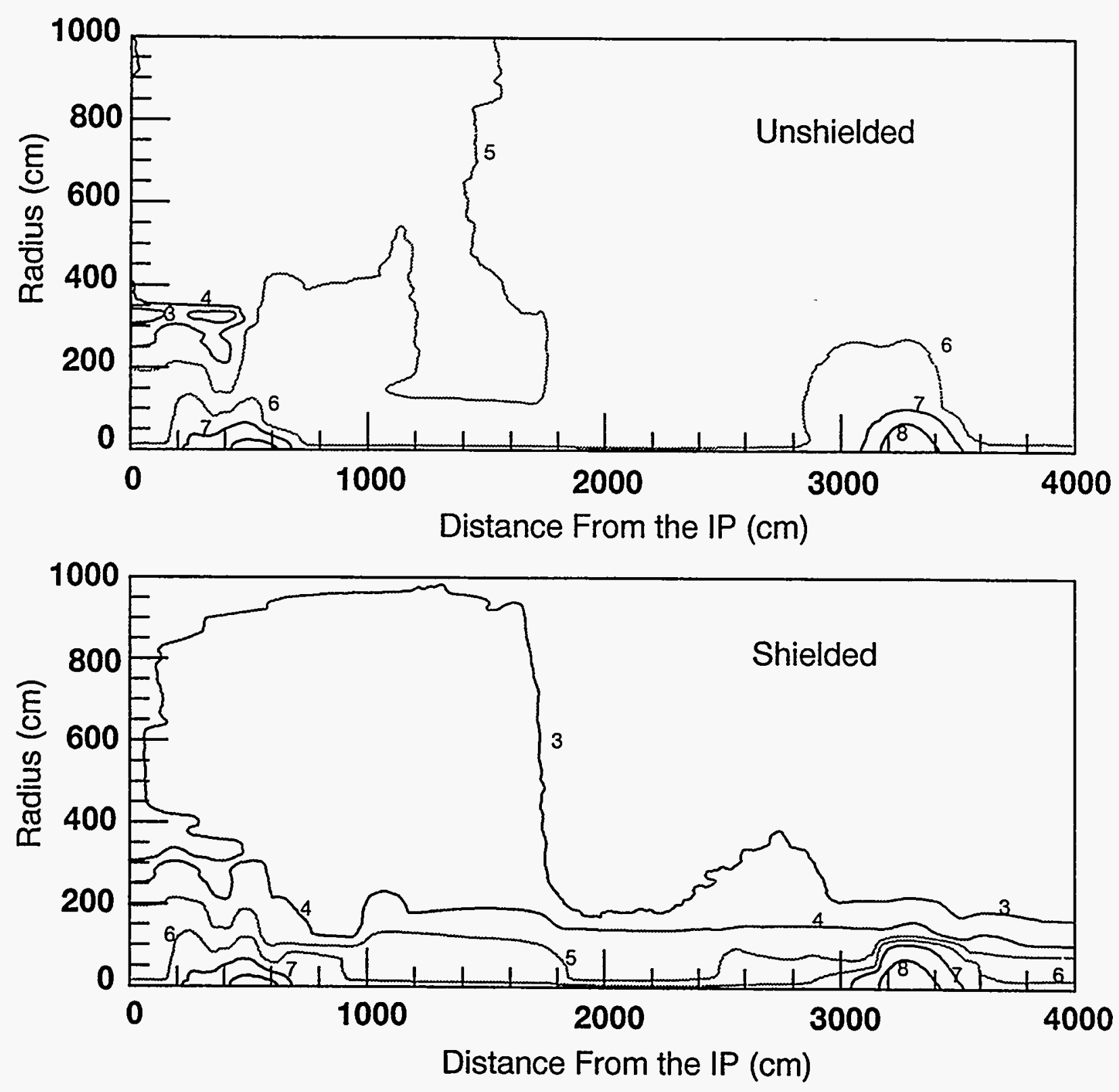

Figure 6 

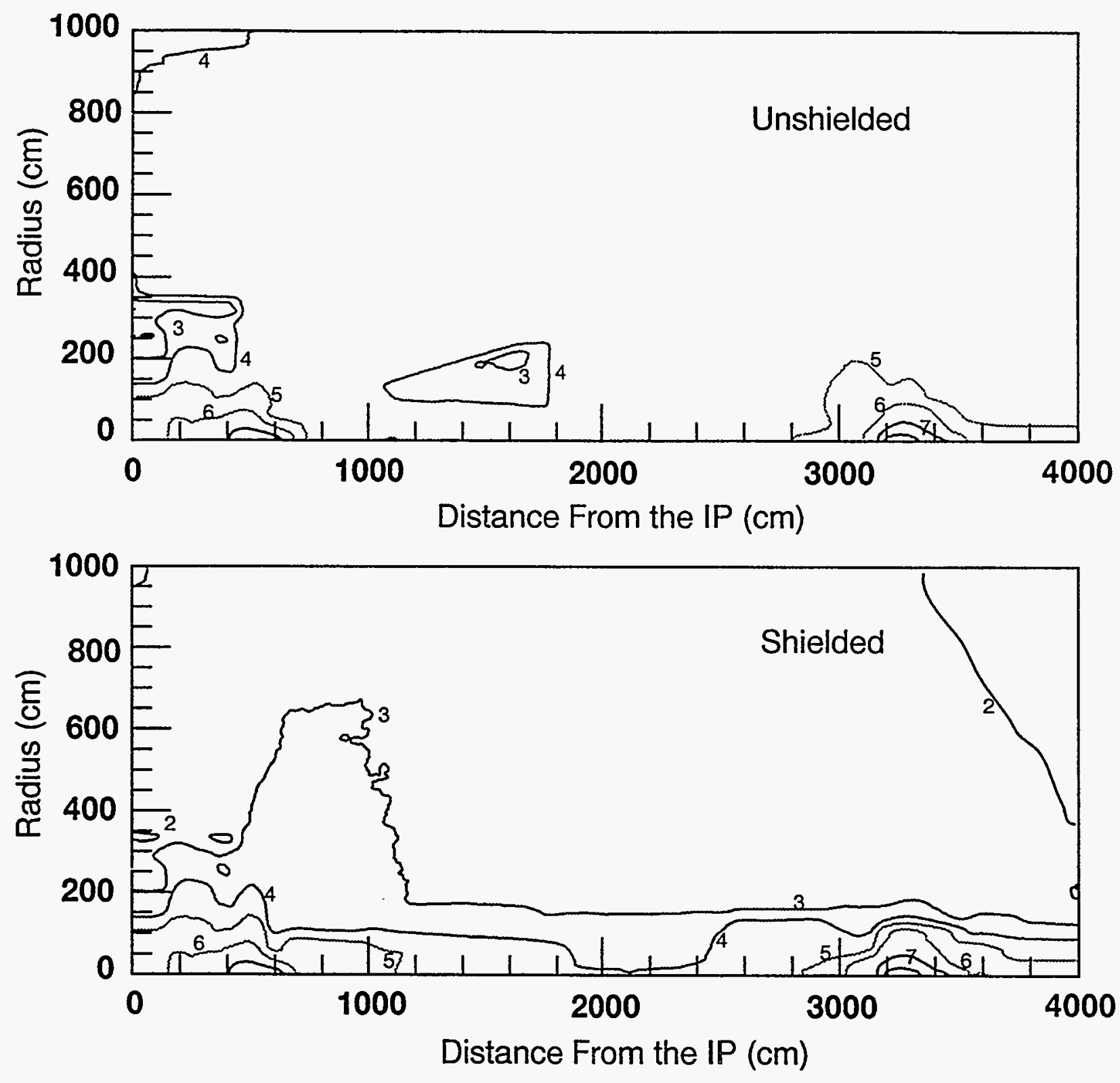

Figure 7 

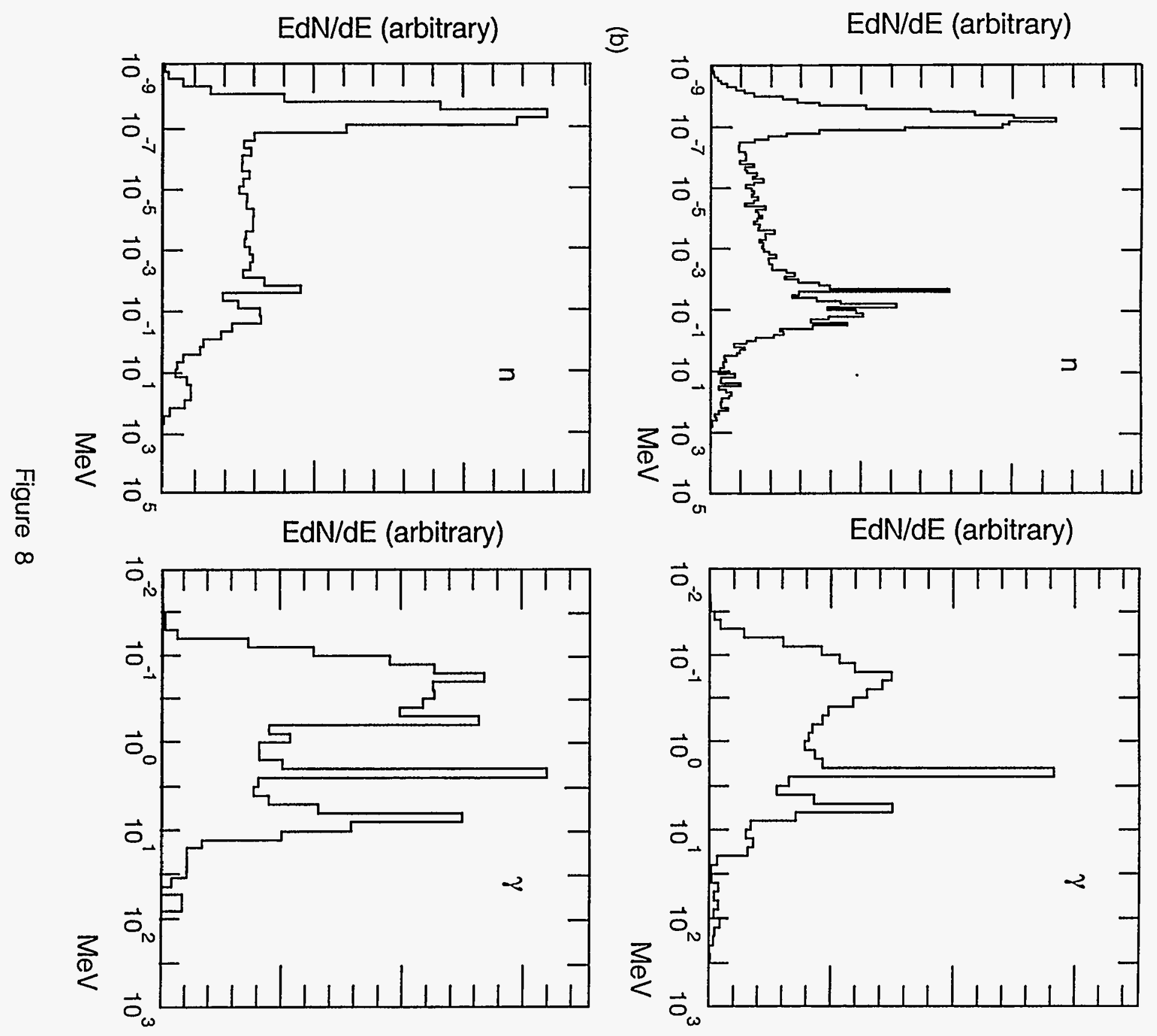

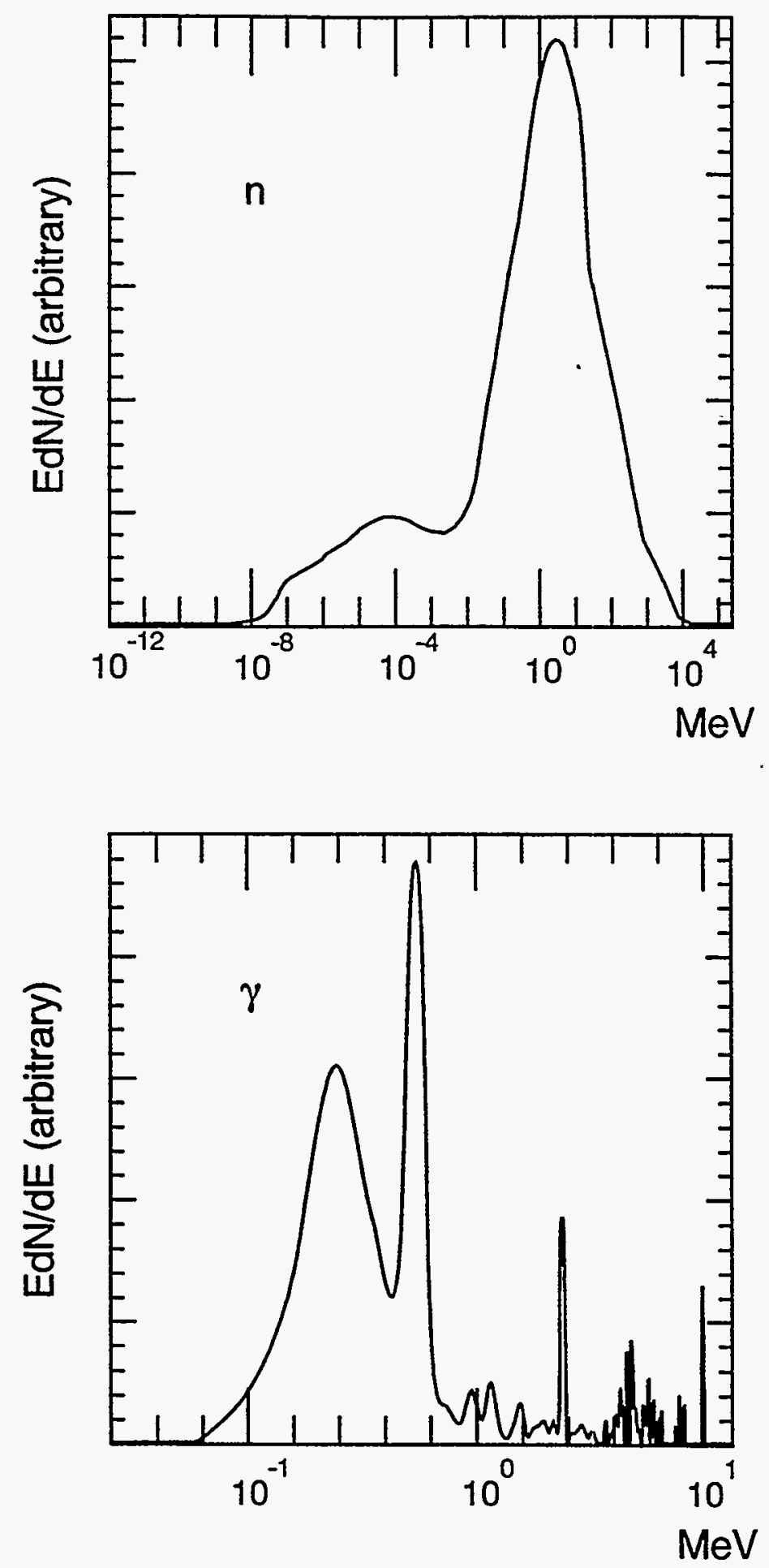

Figure 9 